\title{
NEW STACKED CENTRAL CONFIGURATIONS FOR THE PLANAR 5-BODY PROBLEM
}

\author{
JAUME LLIBRE \\ Departament de Matemàtiques, Universitat Autònoma de Barcelona, 08193 \\ Bellaterra, Barcelona, Catalonia, Spain, \\ e-mail: jllibre@mat.uab.cat, Tel.3493 5811303, Fax.34935812790
}

LUIS FERNANDO MELLO

Instituto de Ciências Exatas, Universidade Federal de Itajubá, Av. BPS 1303,

Pinheirinho, CEP 37.500-903, Itajubá, MG, Brazil,

e-mail:lfmelo@unifei.edu.br

ERNESTO PEREZ-CHAVELA

Departamento de Matemáticas, UAM-Iztapalapa, A.P. 55534, 09340 Iztapalapa, Mexico, D.F., Mexico,

e-mail: epc@xanum.uam.mx

\begin{abstract}
A stacked central configuration in the $n$-body problem is one that has a proper subset of the $n$-bodies forming a central configuration. In this paper we study the case where three bodies with masses $m_{1}, m_{2}, m_{3}$ (bodies $1,2,3$ ) form an equilateral central configuration, and the other two with masses $m_{4}, m_{5}$ are symmetric with respect to the mediatrix of the segment joining 1 and 2 , and they are above the triangle generated by $\{1,2,3\}$. We show the existence and non-existence of this kind of stacked central configurations for the planar 5-body problem.
\end{abstract}

Keywords: Planar central configurations, $n$-body problem, stacked central configurations.

\section{Introduction}

The classical planar Newtonian $n$-body problem in celestial mechanics consists in studying the motion of $n$ pointlike masses in a fixed plane, interacting among themselves through no other forces than their mutual gravitational attraction according to Newton's gravitational law (Newton 1687).

The center of mass of the system, given by $\sum_{j=1}^{n} m_{j} r_{j} / M$, where $M=$ $m_{1}+\cdots+m_{n}$ is the total mass and $r_{j}$ is the position vector of the mass $m_{j}$, is considered at the origin of an inertial system. Usually this inertial system is called the inertial barycentric system.

The simplest motion of the $n$ bodies, called a homographic solution, is a motion such that the configuration of the $n$ bodies remains the 
same (with respect to the inertial barycentric system) up to a dilation and a rotation of $\mathbb{R}^{2}$.

The first homographic solutions for the 3-body problem were found by Euler (1767), for which three bodies are collinear at any time, and by Lagrange (1873), where the three bodies are at any time at the vertices of an equilateral triangle.

At a given instant $t=t_{0}$ the configuration of the $n$ bodies is central if the gravitational acceleration acting on every mass point is proportional to its position (referred to the inertial barycentric system). Central configurations and homographic solutions are linked by the Laplace theorem (see for instance Boccaletti and Pucacco 1996; Wintner 1941): the configuration of the $n$ bodies in a homographic solution is central at any instant of time.

If we have a central configuration, any dilation and any rotation (centered at the center of mass) of it provides another central configuration. We say that two central configurations are similar if we can pass from one to another through a dilation and a rotation. So we can study the classes of central configurations defined by the above equivalence relation. Thus the 3 -body problem has exactly 5 classes of central configurations for any value of the positive masses.

Central configurations of the $n$-body problem are important because: they allow the computation of homographic solutions; if the $n$ bodies are heading for a simultaneous collision then the bodies tend to a central configuration (see Saari 1980); there is a relation between central configurations and the bifurcations of the hypersurfaces of constant energy and angular momentum (see Smale 1970). See also the Refs. Moeckel 1990; Moulton 1910.

The main general open problem for the planar central configurations is due to Wintner (1941) and Smale (1998): Is the number of classes of planar central configurations finite for any choice of the (positive) masses $m_{1}, \ldots, m_{n}$ ?

Hampton and Moeckel in (2006), proved this conjecture for the 4body problem. The conjecture remains open for $n>4$. But if one mass can be negative, Roberts (1999), proved that there exists a oneparameter not equivalent family of planar central configurations for the 5 -body problem. Also considering the particles endowed with masses and charges, Alfaro and Perez-Chavela (2002) proved the existence of a continuum of central configurations in a particular 4-body problem. Other recent papers on central configurations are due to Corbera, Cors and Llibre (2010), Corbera and Llibre (2010), Gidea and Llibre (2010), Piña and Lonngi (2010), ... 
In 2005 Hampton (2005) provides a new family of planar central configurations, called stacked central configuration, for the 5-body problem with an interesting property: the central configuration has a subset of three bodies forming a central configuration of the 3-body problem, in fact these three bodies are in an equilateral triangle, and the remaining two bodies are in the interior of the triangle and are located symmetrically with respect to a perpendicular bisector.

Recently the first and second author of this paper gave new examples of stacked central configurations of the 5-bodies which, as the ones studied by Hampton (2005), have three bodies in the vertices of an equilateral triangle, but the other two are on the perpendicular bisector (Llibre and Mello 2008).

In this paper we find a new class of stacked central configurations in a 5 -body problem which has three bodies in the vertices of an equilateral triangle and the other two are located symmetrically with respect to a perpendicular bisector in the exterior and above of the triangle, see Figure 1.

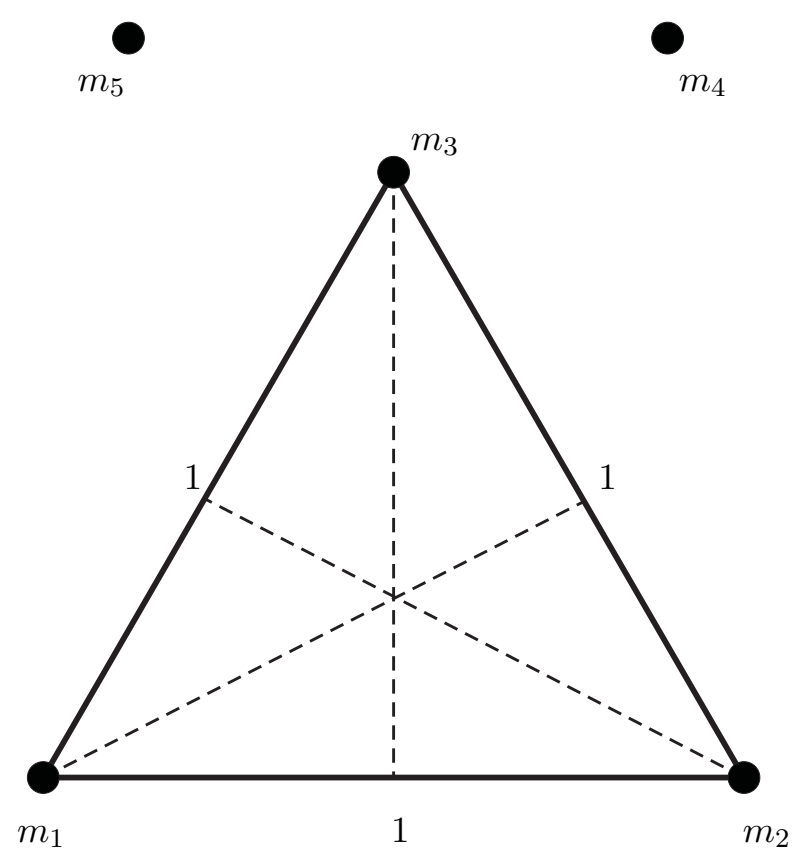

Figure 1. Three bodies at the vertices of an equilateral triangle and two bodies located symmetrically with respect to a perpendicular bisector.

The paper is organized as follows, in Section 2 we prove the existence and non-existence of this new class of stacked central configurations. 
In section 3 we show some numerical evidence of the existence and non-existence of another families of this kind of central configurations.

\section{Existence and non-existence of stacked central configurations}

The equations of motion of the planar Newtonian $n$-body problem are given by

$$
\ddot{r}_{i}=-\sum_{\substack{j=1 \\ j \neq i}}^{n} m_{j} \frac{r_{i}-r_{j}}{r_{i j}^{3}},
$$

for $i=1,2, \ldots, n$. Here the gravitational constant is taken equal to 1 , $r_{j} \in \mathbb{R}^{2}$ is the position vector of the punctual mass $m_{j}$ in the inertial barycentric system, and as before $r_{i j}=\left|r_{i}-r_{j}\right|$ is the Euclidean distance between $r_{i}$ and $r_{j}$.

For the central configurations we have $\ddot{r}_{j}=\lambda r_{j}$ with $\lambda \neq 0$ for all $j=1, \ldots, n$. So from equation (1) we have

$$
\lambda r_{i}=-\sum_{\substack{j=1 \\ j \neq i}}^{n} m_{j} \frac{r_{i}-r_{j}}{r_{i j}^{3}}
$$

for $i=1,2, \ldots, n$.

For the planar central configurations instead of working with equation (2) we shall use the Dziobek equations (see Hagihara 1970, p. 241)

$$
f_{i j}=\sum_{\substack{k=1 \\ k \neq i, j}}^{n} m_{k}\left(R_{i k}-R_{j k}\right) \Delta_{i j k}=0,
$$

for $1 \leq i<j \leq n$, where $R_{i j}=1 / r_{i j}^{3}$ and $\Delta_{i j k}=\left(r_{i}-r_{j}\right) \wedge\left(r_{i}-r_{k}\right)$. As usual $\wedge$ denotes the cross product of two vectors.

For the 5-body problem equations (3) is a set of ten equations. Our class of configurations with five bodies as in Figure 1 without collisions must satisfy

$$
\begin{gathered}
r_{12}=r_{23}=r_{13}=1, \quad r_{14}=r_{25}, \quad r_{15}=r_{24}, \quad r_{34}=r_{35}, \quad r_{14}>r_{24}, \\
\Delta_{124}=\Delta_{125}, \quad \Delta_{143}=\Delta_{235}, \quad \Delta_{145}=\Delta_{245}, \quad \Delta_{135}=\Delta_{243} .
\end{gathered}
$$

We are also assuming $\Delta_{145} \neq 0$, that is, the bodies $m_{1}, m_{4}$ and $m_{5}$ are not on the same straight line. 
The equation $f_{45}=0$ of (3) becomes

$$
\left(R_{14}-R_{15}\right) \Delta_{145}\left(m_{1}-m_{2}\right)=0 .
$$

Thus $m_{1}=m_{2}$. Similarly the equation $f_{12}=0$ of (3) goes over to

$$
\left(R_{14}-R_{24}\right) \Delta_{124}\left(m_{4}-m_{5}\right)=0 .
$$

Therefore $m_{4}=m_{5}$. Substituting $m_{1}=m_{2}$ and $m_{4}=m_{5}$ into the other eight equations of (3) it follows that

$$
\begin{aligned}
& f_{13}=0 \Leftrightarrow f_{23}=0, \quad f_{15}=0 \Leftrightarrow f_{24}=0, \\
& f_{14}=0 \Leftrightarrow f_{25}=0, \quad f_{34}=0 \Leftrightarrow f_{35}=0,
\end{aligned}
$$

which can be written respectively as

$$
\begin{aligned}
L=\left(R_{14}-R_{34}\right) \Delta_{134}+\left(R_{15}-R_{34}\right) \Delta_{135} & =0, \\
\left(R_{12}-R_{15}\right) \Delta_{142} m_{1}+\left(R_{12}-R_{34}\right) \Delta_{143} m_{3}+\left(R_{15}-R_{45}\right) \Delta_{145} m_{4} & =0 \\
\left(R_{12}-R_{14}\right) \Delta_{142} m_{1}+\left(R_{12}-R_{34}\right) \Delta_{153} m_{3}+\left(R_{14}-R_{45}\right) \Delta_{154} m_{4} & =0 \\
{\left[\left(R_{12}-R_{14}\right) \Delta_{134}+\left(R_{12}-R_{15}\right) \Delta_{153}\right] m_{1}+\left(R_{34}-R_{45}\right) \Delta_{345} m_{4} } & =0 .
\end{aligned}
$$

Denote by $A=\left[a_{i j}\right]$ the matrix of the coefficients of the homogeneous linear system in the variables $m_{1}, m_{3}$ and $m_{4}$ defined by equations (5), (6) and (7). Thus

$$
\begin{aligned}
& a_{11}=\left(R_{12}-R_{15}\right) \Delta_{142}, \quad a_{12}=\left(R_{12}-R_{34}\right) \Delta_{143}, \quad a_{13}=\left(R_{15}-R_{45}\right) \Delta_{145}, \\
& a_{21}=\left(R_{12}-R_{14}\right) \Delta_{142}, \quad a_{22}=\left(R_{12}-R_{34}\right) \Delta_{153}, \quad a_{23}=\left(R_{14}-R_{45}\right) \Delta_{154}, \\
& a_{31}=\left(R_{12}-R_{14}\right) \Delta_{134}+\left(R_{12}-R_{15}\right) \Delta_{153}, \quad a_{32}=0, \quad a_{33}=\left(R_{34}-R_{45}\right) \Delta_{345} .
\end{aligned}
$$

In order to have a solutions different from $m_{1}=m_{3}=m_{4}=0$ the determinant of the matrix $A$ must be zero, where

$$
\operatorname{det} A=\left(R_{12}-R_{34}\right)\left[\left(R_{12}-R_{15}\right) \Delta_{153}-\left(R_{12}-R_{14}\right) \Delta_{143}\right] \Gamma,
$$

with $\Gamma$ equal to

$$
\left(R_{34}-R_{45}\right) \Delta_{142} \Delta_{345}+\left[\left(R_{14}-R_{45}\right) \Delta_{143}+\left(R_{15}-R_{45}\right) \Delta_{153}\right] \Delta_{154} .
$$

From equation (4) one has $R_{14} \Delta_{134}=R_{34} \Delta_{134}+\left(R_{34}-R_{15}\right) \Delta_{135}$. Substituting this equation into equation (9) it follows that $\Gamma=0$ and therefore, from (8), we get $\operatorname{det} A=0$. So the homogeneous linear system in the variables $m_{1}, m_{3}$ and $m_{4}$, defined by equations (5), (6) and (7), has nontrivial solutions under the assumption $L=0$, which can be viewed as a constraint on the geometry of the configuration. 


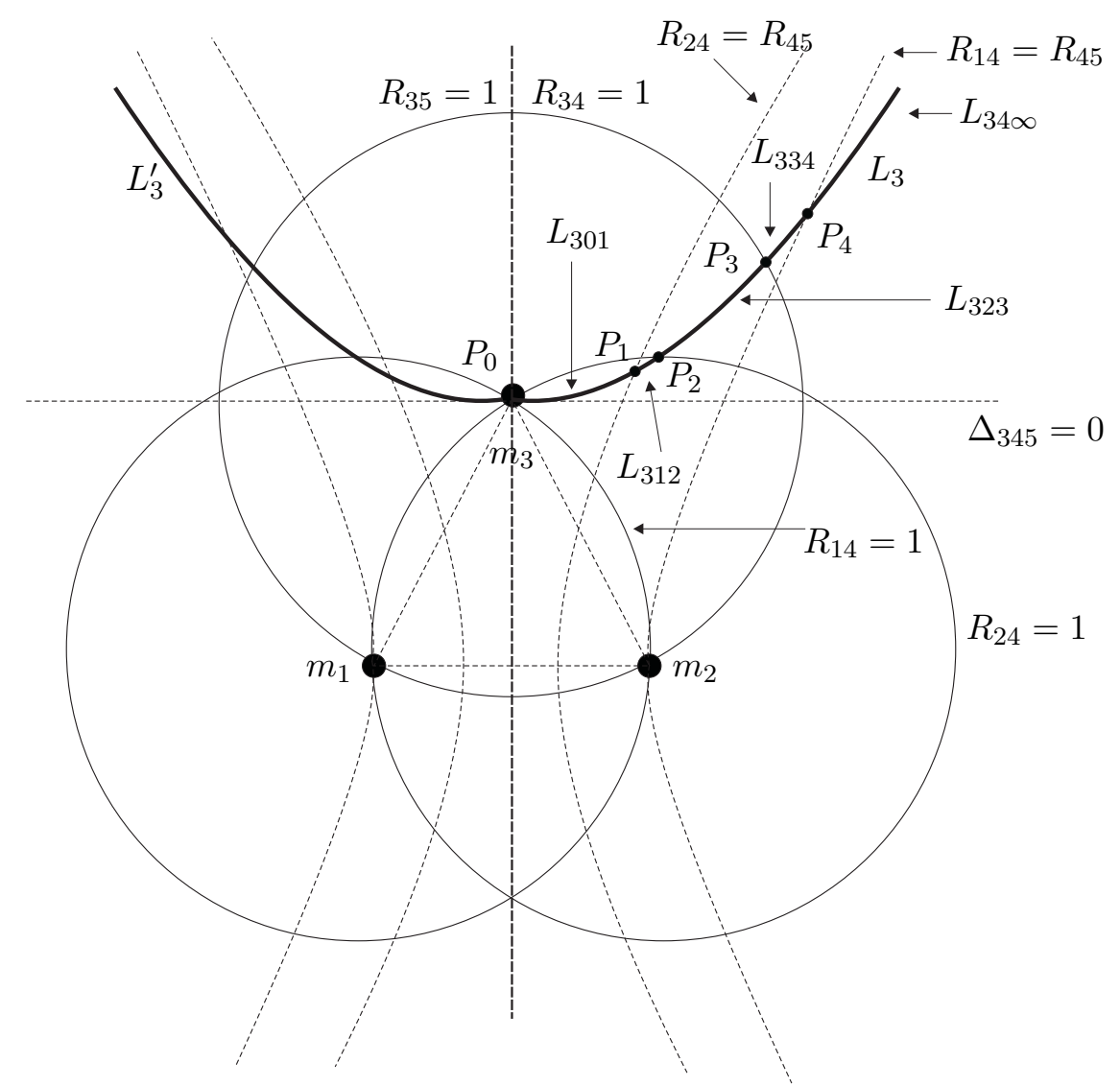

Figure 2. The component of the curve $\{L=0\}$ in the upper semiplane and its components $L_{3}$ and $L_{3}^{\prime}$.

LEMMA 1. Consider the regions

$$
\begin{gathered}
S_{1}=\left\{R_{45}>R_{34}>R_{14}>1\right\}, \quad S_{2}=\left\{1>R_{14}>R_{34}>R_{45}\right\}, \\
S_{3}=\left\{\Delta_{345}>0, \Delta_{134}<0, R_{34}>R_{24}\right\}
\end{gathered}
$$

and $S_{1}^{\prime}, S_{2}^{\prime}, S_{3}^{\prime}$ the symmetrical regions with respect to the mediatrix of the segment joining $r_{1}$ and $r_{2}$. See Figure 3. The curves

$$
L_{i}=\{L=0\} \cap S_{i}, \quad L_{i}^{\prime}=\{L=0\} \cap S_{i}^{\prime},
$$

$i=1,2,3$, are well-defined and are not empty. See Figure 2.

Proof. Without loss of generality we can take a coordinate system such that $r_{1}=(-1 / 2,0), r_{2}=(1 / 2,0)$ and $r_{3}=(0, \sqrt{3} / 2)$ respectively. 
Thus $r_{4}=(x, y)$ and $r_{5}=(-x, y)$, where $x>0$ and $y \in \mathbb{R}$. With these coordinates we have

$$
\begin{aligned}
L(x, y) & =\left[\frac{1}{\left((x+1 / 2)^{2}+y^{2}\right)^{3 / 2}}-\frac{1}{\left(x^{2}+(y-\sqrt{2} / 2)^{2}\right)^{3 / 2}}\right] M_{1} \\
& +\left[\frac{1}{\left((x-1 / 2)^{2}+y^{2}\right)^{3 / 2}}-\frac{1}{\left(x^{2}+(y-\sqrt{2} / 2)^{2}\right)^{3 / 2}}\right] M_{2},
\end{aligned}
$$

where $M_{1}=\frac{y}{2}-\frac{x \sqrt{3}}{2}-\frac{\sqrt{3}}{2}$ and $M_{2}=\frac{y}{2}+\frac{\sqrt{3} x}{2}-\frac{\sqrt{3}}{2}$. It is easy to see from the above equation that the curve $L(x, y)=0$ is symmetric with respect to the mediatrix of the segment joining $r_{1}$ and $r_{2}$. Using Mathematica we have plotted the graph of the curve $L(x, y)=0$, see Figure 2.

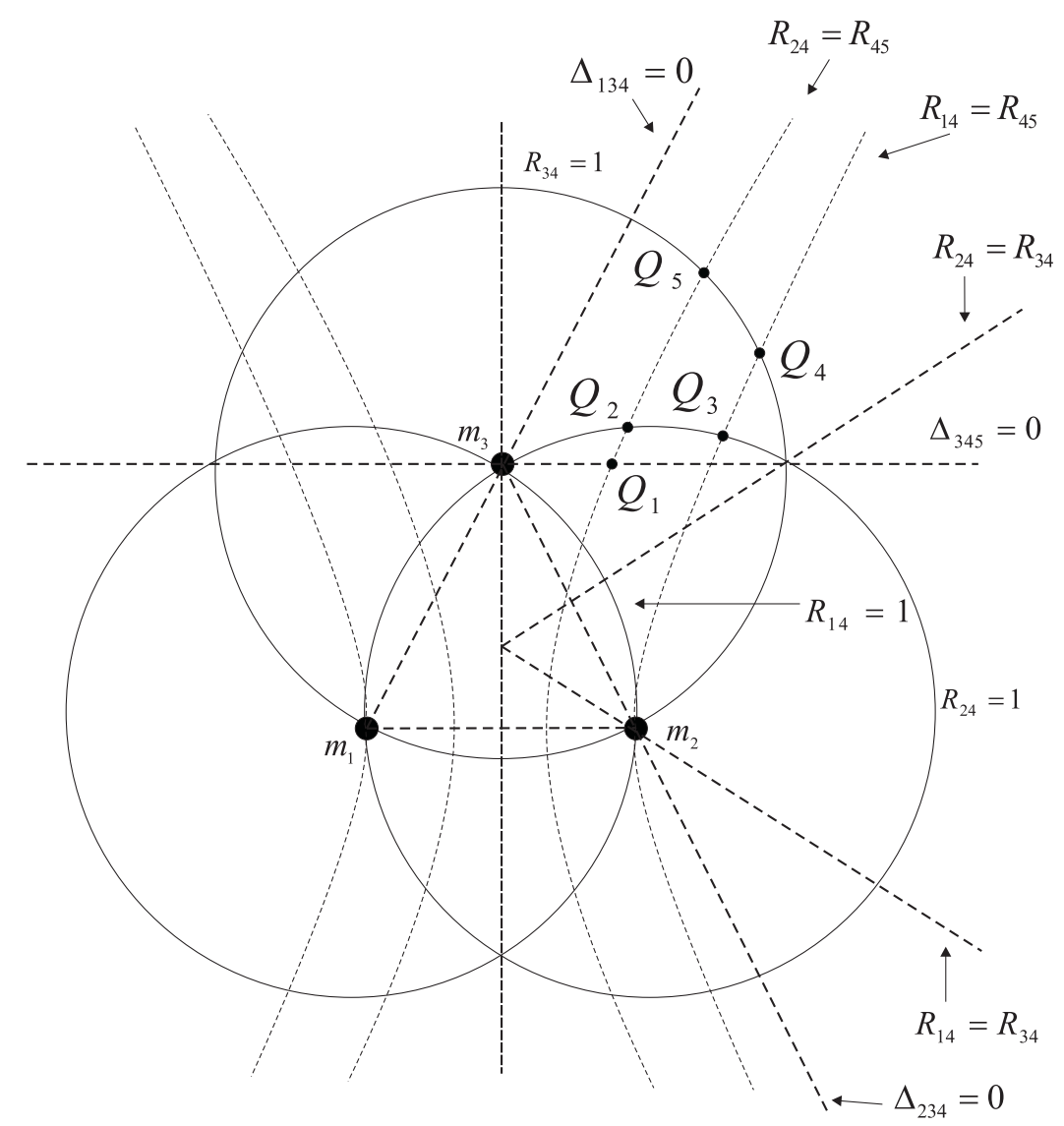

Figure 3. Some straight lines and curves for the calculation of the sign of $L$. 
Define the points

$$
\begin{aligned}
& Q_{1}=\left\{\Delta_{345}=0\right\} \cap\left\{R_{24}=R_{45}\right\} \cap \overline{S_{3},} \\
& Q_{2}=\left\{R_{24}=1\right\} \cap\left\{R_{24}=R_{45}\right\} \cap S_{3}, \\
& Q_{3}=\left\{R_{24}=1\right\} \cap\left\{R_{14}=R_{45}\right\} \cap S_{3}, \\
& Q_{4}=\left\{R_{34}=1\right\} \cap\left\{R_{14}=R_{45}\right\} \cap S_{3}, \\
& Q_{5}=\left\{R_{34}=1\right\} \cap\left\{R_{24}=R_{45}\right\} \cap S_{3},
\end{aligned}
$$

according to Figure 3. The symmetrical points are denoted by $Q_{i}^{\prime}$, $i=1,2,3,4,5$. In (Hampton 2005) Hampton studied stacked central configurations when $r_{4}$ and $r_{5}$ are on the components $L_{1}$ and $L_{1}^{\prime}$ respectively. If $r_{4} \in\left\{\Delta_{234}=0\right\}$ (see Figure 3) then $r_{5} \in\left\{\Delta_{135}=0\right\}$. Thus $L=\left(R_{14}-R_{34}\right) \Delta_{134}<0$. If $r_{4} \in\left\{R_{14}=R_{34}\right\}$ (see Figure 3 ) then $L=\left(R_{15}-R_{34}\right) \Delta_{135}>0$. Therefore there is a connected component of $L=0$ between the lines $\left\{\Delta_{234}=0\right\}$ and $\left\{R_{14}=R_{34}\right\}$ denoted by $L_{2}$. See Figure 2. Its symmetrical component is denoted by $L_{2}^{\prime}$.

If $r_{4} \in\left\{\Delta_{134}=0\right\}$ (see Figure 3) then $L=\left(R_{24}-R_{34}\right) \Delta_{135}<0$. If $r_{4} \in\left\{R_{24}=R_{34}\right\}$ (see Figure 3) then $L=\left(R_{14}-R_{34}\right) \Delta_{134}>0$. By the same argument $L>0$ on the set $\left\{\Delta_{345}=0\right\}$. Therefore there is a connected component of $L=0$ between the lines $\left\{\Delta_{134}=0\right\}$ and $\left\{R_{24}=R_{34}\right\} \cup\left\{\Delta_{345}=0\right\}$ denoted by $L_{3}$. See Figure 2. Its symmetrical component is denoted by $L_{3}^{\prime}$.

In the coordinate system that we have introduced previously one has

$$
\begin{gathered}
Q_{1}=\left(\frac{\sqrt{13}-1}{6}, \frac{\sqrt{3}}{2}\right), Q_{2}=\left(\frac{1}{2}, 1\right), Q_{3}=\left(\frac{1+\sqrt{5}}{4}, \sqrt{1-\left(\frac{\sqrt{5}-1}{4}\right)^{2}}\right), \\
Q_{4}=\left(x_{4}, y_{4}\right), \quad Q_{5}=\left(x_{5}, y_{5}\right),
\end{gathered}
$$

where

$$
\begin{gathered}
x_{4}=\frac{1}{4}(\sqrt{3} \cos (\pi / 18)+2 \cos (\pi / 9)+\sin (\pi / 18)), \\
y_{4}=\frac{1}{2}(\cos (\pi / 18)-\sqrt{3}(\sin (\pi / 18)-1)), \\
x_{5}=\frac{1}{2} \sqrt{2+\cos (\pi / 9)-\sqrt{3} \sin (\pi / 9)}, \\
y_{5}=\frac{1}{2}(\cos (\pi / 18)+\sqrt{3}(1+\sin (\pi / 18))) .
\end{gathered}
$$

From elementary calculations one has the following things for $L: L\left(Q_{1}\right)>$ $0, L\left(Q_{2}\right)<0, L\left(Q_{3}\right)>0, L\left(Q_{4}\right)>0$ and $L\left(Q_{5}\right)<0$. 
Since $Q_{2}, Q_{3}$ are on the circle $R_{24}=1$ and $L\left(Q_{2}\right)<0, L\left(Q_{3}\right)>0$, by continuity there exists at least one point in the intersection of the curve $L_{3}$ with the circle $R_{24}=1$. There is numerical evidence that this point is unique, we denote it as $P_{2}$. Let be $P_{2}^{\prime}$ be the symmetric point respect to the mediatrix of the segment joining the particle with mass $m_{1}$ with the particle with mass $m_{2}$.

The main result of this paper is the following.

THEOREM 2. Assume that the points $r_{1}, r_{2}$ and $r_{3}$ (with positive masses $m_{1}, m_{2}$ and $m_{3}$ ) are at the vertices of an equilateral triangle, whose sides have length 1 , and the points $r_{4}$ and $r_{5}$ (with masses $m_{4}$ and $m_{5}$ ) are located symmetrically with respect to a perpendicular bisector, as in Figure 1. Then there exist stacked central configurations for which the particles with masses $m_{4}$ and $m_{5}$ are in a small arc of $L_{3}$ near the points $P_{2}$ and $P_{2}^{\prime}$ respectively.

Proof. When the particles 4 and 5 are at $P_{2}$ and $P_{2}^{\prime}$ respectively, then since $R_{15}=R_{24}=1$ we obtain $a_{31}=\left(1-R_{14}\right) \Delta_{134}<0$. Therefore the signs of the coefficients of the matrix $A$ are given by

$$
\left.A\right|_{P_{2} \cup P_{2}^{\prime}}=\left(\begin{array}{ccc}
0 & - & + \\
- & + & + \\
- & 0 & +
\end{array}\right) .
$$

By the first row of the above matrix the masses $m_{3}$ and $m_{4}$ have the same sign and by the last row of the matrix the masses $m_{1}$ and $m_{4}$ also have the same sign. So the masses $m_{1}, m_{3}$ and $m_{4}$ have the same sign. Hence when the particles 4 and 5 are at $P_{2}$ and $P_{2}^{\prime}$ respectively, there exists masses $m_{1}, m_{3}$ and $m_{4}$ which form a stacked central configuration.

Now, since $a_{31}$ depends continuously on the mutual distances, we assure that $a_{31}<0$ when particles 4 and 5 are in a small neighborhood of $P_{2}$ and $P_{2}^{\prime}$ respectively, the same is true for the other coefficients of $a_{i j}$, which preserve its sign in a small neighborhood of $P_{2}$ and $P_{2}^{\prime}$. Using the constraint $L=0$ we obtain the small arc cited in the statement of the theorem.

LEMMA 3. The function $a_{31}=\left(R_{12}-R_{14}\right) \Delta_{134}+\left(R_{12}-R_{15}\right) \Delta_{153}$ is negative on the set $\{L=0\} \bigcap\left\{R_{24}<1\right\}$.

Proof. For our class of central configurations one has $R_{14}<R_{24}$. Substituting this inequality into the expression of $a_{31}$ we have $a_{31}<$ $\left(\Delta_{143}+\Delta_{135}\right)\left(R_{24}-1\right)<0$ if $r_{24}>1$. Thus the lemma follows for the components of $L_{3}$ in the exterior of the circle $\left\{R_{24}=1\right\}$. 
Let $P_{0}$ be the vertex of the triangle at $r_{3}$. If the particles 4 and 5 are on the curve $L=0$ and close enough to $P_{0}$, there are no stacked central configurations. In other words we have the following property

PROPOSITION 4. Assume that we have the masses $m_{1}, m_{2}$ and $m_{3}$ at the vertices of an equilateral triangle, whose sides have length 1 , and the masses $m_{4}$ and $m_{5}$ are at $L_{3}=0$ and $L_{3}^{\prime}$ respectively, located symmetrically with respect to a perpendicular bisector. If $r_{4}$ and $r_{5}$ are sufficiently close to $P_{0}$, then there are no positive masses $m_{1}=m_{2}$, $m_{4}=m_{5}$ and $m_{3}$ such that the five bodies with these masses form stacked central configurations.

Proof. With the hypothesis of the proposition, we have $\Delta_{142}<0$, by the other hand the point $r_{4}$ is inside the circle $R_{24}=1$ which corresponds to the region $R_{24}>1$, that is $R_{24}=R_{15}<1$, therefore $a_{11}=\left(R_{12}-R_{15}\right) \Delta_{142}<0$ in this region and the signs of the coefficients of the matrix $A$ are given by

$$
A=\left(\begin{array}{ccc}
- & - & - \\
- & + & + \\
a_{31} & 0 & +
\end{array}\right) .
$$

The first row in the above matrix implies that $m_{1}=m_{2}, m_{4}=m_{5}$ and $m_{3}$ cannot have the same sign.

\section{Numerical evidence of new stacked central configurations}

There is numerical evidence that $L_{3}$ intersects the hyperbola $R_{14}=R_{45}$ at a unique point denoted by $P_{4}$. Also based on numerical evidences we define $L_{34 \infty}$ to be the unbounded connected component of $L_{3}$ with one endpoint at $P_{4}$. As usual, $L_{34 \infty}^{\prime}$ is the unbounded connected component of $L_{3}^{\prime}$ with one endpoint at $P_{4}^{\prime}$.

LEMMA 5. There are no stacked central configurations when $r_{4}$ and $r_{5}$ are on $L_{34 \infty}$ and $L_{34 \infty}^{\prime}$, respectively.

Proof. When $r_{4}$ and $r_{5}$ are at $P_{4}$ and $P_{4}^{\prime}$, respectively, it is easy verify that $a_{21}<0, a_{22}<0$ and $a_{23}=0$ and this implies that the masses $m_{1}$ and $m_{3}$ have opposite signs. 
On the other hand, if $r_{4}$ and $r_{5}$ are on the open $\operatorname{arcs} L_{34 \infty}$ and $L_{34 \infty}^{\prime}$, respectively, we have that $a_{21}<0, a_{22}<0$ and $a_{23}<0$ and this implies that two of the masses $m_{1}, m_{3}$ and $m_{4}$ have opposite signs. This proves (5).

There is numerical evidence that $L_{3}$ intersects the hyperbola $R_{24}=$ $R_{45}$ in a unique point denoted as $P_{1}$, and intersects the circle $R_{34}=1$ in a unique point denoted as $P_{3}$. We now define: $L_{312}, L_{323}$ and $L_{334}$ be the open $\operatorname{arcs} L_{3 i j}$ of $L_{3}$, whose endpoints are $P_{i}$ and $P_{j}$, for $i, j=1,3,4$. More precisely,

$$
\begin{gathered}
L_{312}=L_{3} \cap\left\{\Delta_{345}>0, R_{24}>1, R_{24}>R_{45}, R_{14}<R_{45}\right\}, \\
L_{323}=L_{3} \cap\left\{R_{24}<1, R_{24}>R_{45}, R_{14}<R_{45}, R_{34}>1\right\}, \\
L_{334}=L_{3} \cap\left\{R_{34}<1, R_{24}>R_{45}, R_{14}<R_{45}\right\} .
\end{gathered}
$$

The same definitions are valid for $L_{3}^{\prime}$, see Figure 2 .

In order to give numerical evidence for the existence of new stacked central configurations, we have calculated the coordinates of the points $P_{i}, i=1,2,3,4$

$$
\begin{aligned}
& P_{1}=(0.45179079888471224,0.9022946174921869), \\
& P_{2}=(0.63217335448833913,0.9912266160486711), \\
& P_{3}=(0.93395902567645331,1.2234055072562044), \\
& P_{4}=(0.95907733939463801,1.2451548857175381) .
\end{aligned}
$$

LEMMA 6. There are no stacked central configurations when $r_{4}$ and $r_{5}$ are on the components $L_{334}$ and $L_{334}^{\prime}$, respectively.

Proof. For $r_{4} \in L_{334}$ and $r_{5} \in L_{334}^{\prime}$ the signs of the coefficients of the matrix $A$ are given by

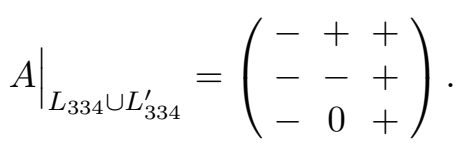

Using the last row of the above matrix we conclude that $m_{1}$ and $m_{4}$ have the same sign. Unfortunately we cannot obtain more information about the sign of the mass $m_{3}$ from the above matrix. Nevertheless, equations (5) and (7) define two planes through the origin in the space $\left(m_{1}, m_{3}, m_{4}\right)$. The normal vectors of these planes are, respectively, $n_{1}=$ 
$\left(a_{11}, a_{12}, a_{13}\right)$ and $n_{3}=\left(a_{31}, 0, a_{33}\right)$. Let $T=\left(T_{1}, T_{2}, T_{3}\right)=n_{1} \wedge n_{3}$ be the vector parallel to the straight line defined by the intersection of these two planes. Thus there exist positive masses $m_{1}, m_{3}$ and $m_{4}$ as solutions of equations (5) and (7) if and only if the components of the vector $T$ have the same sign. It is easy verify that

$$
T_{1}=a_{12} a_{33}>0, \quad \text { and } \quad T_{3}=-a_{12} a_{31}>0 .
$$

In order to see that $T_{2}=a_{13} a_{31}-a_{11} a_{33}<0$, and since we already know the coordinates of the points $P_{3}$ and $P_{4}$, we can verify numerically that the values of $T_{2}$ on the curve $L=0$ between the points $P_{3}$ and $P_{4}$ are in the interval $(-0.376045,-0.368863)$. As not all the above components have the same sign, Lemma 6 has been proved.

LEMMA 7. When $r_{4}$ and $r_{5}$ are at $P_{3}$ and $P_{3}^{\prime}$, respectively, there are no stacked central configurations.

Proof. When $r_{4}$ and $r_{5}$ are at $P_{3}$ and $P_{3}^{\prime}$, the matrix $A$ takes the form

$$
A=\left(\begin{array}{ccc}
-0.664093 & 0 & 0.694115 \\
-1.04073 & 0 & 0.00940803 \\
-1.0721 & 0 & 0.56513
\end{array}\right) .
$$

Then, since columns 1 and 3 in the above matrix are linearly independent the masses $m_{1}$ and $m_{4}$ must vanish. Therefore there are no stacked central configurations in this case.

The next proposition brings numerical evidence of the existence of stacked central configurations for $r_{4} \in L_{323}$.

PROPOSITION 8. Assume that $r_{1}, r_{2}$ and $r_{3}$ (with positive masses $m_{1}, m_{2}$ and $m_{3}$ ) are at the vertices of an equilateral triangle whose sides have length $1, r_{4}$ and $r_{5}$ (with positive masses $m_{4}$ and $m_{5}$ ) are located symmetrically with respect to a perpendicular bisector such that $r_{4} \in L_{323}$ and $r_{5} \in L_{323}^{\prime}$, as in Figure 2. Then there are positive masses $m_{1}=m_{2}, m_{4}=m_{5}$ and $m_{3}$ such that the five bodies with these masses form stacked central configurations.

Proof. For $r_{4} \in L_{323}$ and $r_{5} \in L_{323}^{\prime}$ the signs of the coefficients of the matrix $A$ are given by

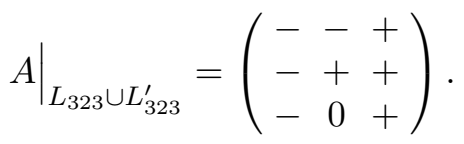


Using the last row of the above matrix we conclude that $m_{1}$ and $m_{4}$ have the same sign. Unfortunately we cannot obtain more information about the sign of the mass $m_{3}$ from the above matrix. Nevertheless, equations (5) and (6) define two planes through the origin in the space $\left(m_{1}, m_{3}, m_{4}\right)$. The normal vectors of these planes are, respectively, $n_{1}=$ $\left(a_{11}, a_{12}, a_{13}\right)$ and $n_{2}=\left(a_{21}, a_{22}, a_{23}\right)$. Let $T=\left(T_{1}, T_{2}, T_{3}\right)=n_{1} \wedge n_{2}$ be the vector parallel to the straight line defined by the intersection of these two planes. Thus there exist positive masses $m_{1}, m_{3}$ and $m_{4}$ as solutions of equations (5) and (6) if and only if the components of the vector $T$ have the same sign. An easy computation shows that

$$
T_{1}=a_{12} a_{23}-a_{13} a_{22}<0, \quad T_{3}=a_{11} a_{22}-a_{12} a_{21}<0 .
$$

In order to see that $T_{2}=a_{13} a_{21}-a_{11} a_{23}<0$, we have verified numerically that the values of $T_{2}$ on the curve $L=0$ between the points $P_{2}$ and $P_{3}$ are in the interval $(-0.716137,-0.44343)$. The Proposition 8 has been proved.

\section{Acknowledgements}

The authors thank to the anonymous referees for their deep review of the original version. Their valuable comments and suggestions helped us to improve this work. The first author is partially supported by a MEC/FEDER grant number MTM2008 03437, by a CIRIT grant number 2009 SGR 410 and by ICREA Academia. The second author developed this work under the project CNPq 473747/2006-5. This work was initiated while the second author visited Universitat Autònoma de Barcelona, supported by CNPq grant 210056/2006-1. The third author has been partially supported by CONACYT-México, grant 12378 .

\section{References}

Alfaro, F. and Perez-Chavela E.: 2002, 'Families of continua of central configurations in charged problems', Dynamics of Continuous, Discrete and Impulsive Systems. Series A: Mathematical Analysis 9, 463-475.

Boccaletti, D. and Pucacco, G.: 1996, Theory of Orbits, Vol. 1, Integrable systems and non-perturbative methods. Astronomy and Astrophysics Library. SpringerVerlag, Berlin.

Corbera, M. and Llibre, J.: 2010, 'On the existence of central configurations of $p$ nested regular polyhedra', Celestial Mechanics and Dynamical Astronomy 106, $197-207$. 
Corbera, M., Cors, J.M. and Llibre, J.: 2010, 'On the central configurations of the planar 1+3 body problem', Celestial Mechanics and Dynamical Astronomy 109, $27-43$.

Euler, L.: 1767, 'De moto rectilineo trium corporum se mutuo attahentium', Novi Comm. Acad. Sci. Imp. Petrop. 11, 144-151.

Gidea,M. and Llibre, J.: 2010, 'Symmetric planar central configurations of five bodies: Euler plus two', Celestial Mechanics and Dynamical Astronomy 106, 89-107.

Hagihara, Y.: 1970, Celestial Mechanics, Vol. 1. MIT Press, Massachusetts.

Hampton, M.: 2005, 'Stacked central configurations: new examples in the planar five-body problem', Nonlinearity 18, 2299-2304.

Hampton, M. and Moeckel, R.: 2006, 'Finiteness of relative equilibria of the fourbody problem', Invent. Math. 163, 289-312.

Lagrange, J.L.: 1873, Essai sur le problème de trois corps, Ouvres, Vol. 6. GauthierVillars, Paris.

Llibre, J. and Mello, L.F.: 2008, 'New central configurations for the planar 5-body problem', Celestial Mech. Dyn. Astr. 100, 141-149.

Moeckel, R.: 1990, 'On central configurations', Math. Z. 205, 499-517.

Moulton, F.R.: 1910, 'The straight line solutions of $n$ bodies', Ann. of Math. 12, $1-17$.

Newton, I.: 1687, Philosophi Naturalis Principia Mathematica. Royal Society, London.

Piña, E., and Lonngi, P.: 2010, 'Central configurations for the planar Newtonian four-body problem', Celestial Mechanics and Dynamical Astronomy 108, 73-93.

Roberts, G.E.: 1999, 'A continuum of relative equilibria in the five-body problem', Physica D 127, 141-145.

Saari, D.: 1980, 'On the role and properties of central configurations', Cel. Mech. 21, 9-20.

Smale, S.: 1970, 'Topology and mechanics II: The planar $n$-body problem', Invent. Math. 11, 45-64.

Smale, S.: 1998, 'Mathematical problems for the next century', Math. Intelligencer 20, 7-15.

Wintner, A.: 1941, The Analytical Foundations of Celestial Mechanics. Princeton University Press. 